\title{
Nausea, vomiting and diarrhea: An unusual presentation of multiple sclerosis
}

\author{
Andrew Szilagyi MD FRCPC, Hyman M Schipper MD FRCPC, Norman Just MD FRCPC
}

\begin{abstract}
A Szilagyi, HM Schipper, N Just. Nausea, vomiting and diarrhea: An unusual presentation of multiple sclerosis. Can J Gastroenterol 1997;11(4):367-370. The case of a young woman who presented with nausea, vomiting and diarrhea is outlined; the etiology turned out to be a first attack of multiple sclerosis. Plausible mechanisms are discussed.
\end{abstract}

Key Words: Diarrhea, Multiple sclerosis, Vomiting

\author{
Nausées, vomissements et diarrhée : tableau \\ inusité de la sclérose en plaques
}

RÉSUMÉ : On présente ici le cas d'une jeune femme présentant des nausées, des vomissements et de la diarrhée. L'étiologie s'est révélée être une première crise de sclérose en plaques. Les mécanismes possiblement en cause sont décrits ici.
$\mathrm{M}$ ultiple sclerosis is a multifocal demyelinating disorder occurring with highest incidence in temperate climate zones. It usually affects young adults with subacute onset of focal neurological symptoms. The majority $(80 \%)$ of patients present with visual, sensory or gait disturbances $(1,2)$, whereas older patients (older than 40 years) more commonly exhibit symptoms of progressive myelopathy (2).

Nausea, vomiting and diarrhea are three of the most common symptoms encountered in patients seen by both family physicians and gastroenterologists. In the vast majority, the causes include specific or nonspecific gastrointestinal infections (3). However this symptom complex can be caused by neurological disease. The usual neurological etiologies include autonomic neuropathies $(4,5)$, but rarely central causes can be seen (6-8). This symptom complex is an unusual presenting feature and may easily confound the diagnosis of multiple sclerosis, particularly if focal central nervous system signs are absent. The case of a young woman whose initial symptoms of nausea, vomiting and diarrhea appear to represent a first attack of multiple sclerosis is presented.

\section{CASE PRESENTATION}

In June 1990 a 33-year old right-handed woman presented to the emergency room with a two-week history of nausea, vomiting and intermittent (one to four times/day) loose watery stools without blood. The vomiting and diarrhea were not associated with cramps, fever or chills. She experienced mild light-headedness, especially on arising, and complained of mild left neck pain. On the day of admission she vomited three times. There were no symptoms of upper respiratory tract infection and no history of travel or contact with subjects with diarrhea. She is married and had two healthy children by caesarian section. She was last treated with antibiotics for sinusitis in 1985. There was no history of alcohol or substance abuse. The patient's mother died of disabling multiple sclerosis, and a younger brother was recently diagnosed with this demyelinating disease.

Physical examination at admission disclosed a thin woman in no acute distress, with normal vital signs and no postural changes. The general and neurological examinations were entirely within normal limits.

Departments of Neurology and Radiology, and Division of Gastroenterology, Sir Mortimer B Davis Jewish General Hospital, McGill University School of Medicine and Faculty of Medicine, Montreal, Quebec

Correspondence and reprints: Dr A Szilagyi, 6000 Côte des Neiges, \#100, Montréal, Québec H3S 128. Telephone 514-739-7370, fax 514-340-8282

Received for publication May 31, 1996. Accepted November 14, 1996 


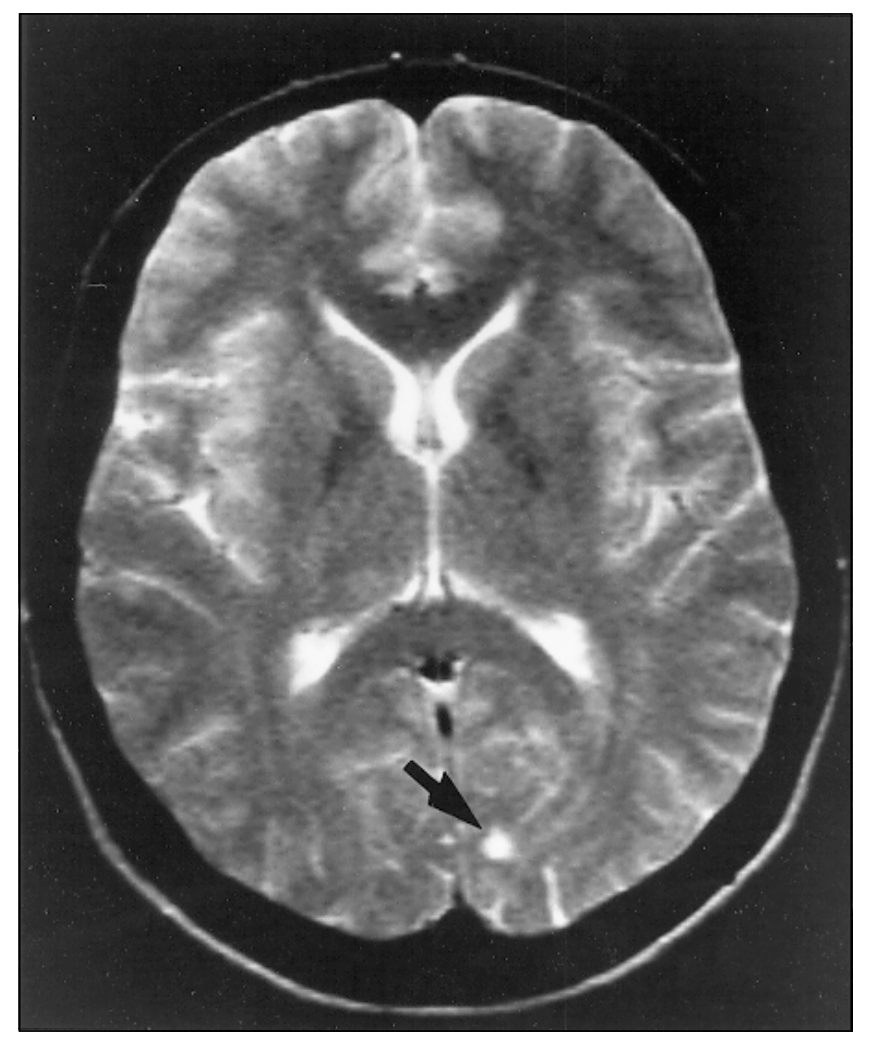

Hematocrit was $36.3 \%$, white blood cell count $5.3 \times 10^{9} / \mathrm{L}$, mean cell volume $85.6 \mathrm{fL}$, blood glucose $4.5 \mathrm{mmol} / \mathrm{L}$, blood urea nitrogen $2.9 \mathrm{mmol} / \mathrm{L}$ and creatinine $84 \mu \mathrm{mol} / \mathrm{L}$. Electrolytes and liver enzymes were normal. A free thyroxine index and thyroid stimulating hormone were normal. Urinalysis and urine cultures were normal. A Venereal Disease Research Laboratory test for syphilis was negative. An electrocardiogram test was normal.

The patient was admitted for further evaluation. A gastroenterological work-up consisting of stool occult blood, cultures, sensitivity, ova and parasites were all negative. Gastroscopy and an upper gastrointestinal small bowel follow-through study were also normal. A colonoscopy to splenic flexure with biopsy of normal-looking mucosa disclosed mild nonspecific edema and occasional inflammatory cells in the rectosigmoid. A liquid gastric emptying scan showed $73 \%$ retention after 30 mins (normal less than $50 \%$ ). A solid gastric emptying scan was normal.

Because symptoms persisted and nausea was a prominent feature, and because of the family history, neurological consultation was obtained. Clinical evaluation and a Bàràny test were normal. A noncontrast computed tomographic (CT) scan of the head revealed a small hypodense lesion in the left subcortical white matter. A follow-up double dose delayed contrast CT scan of the head revealed two diffusely enhancing left parietal white matter lesions compatible with multiple sclerosis. Magnetic resonance imaging (MRI) with $\mathrm{T}_{2}$-weighted images disclosed a much wider involvement of the subcortical white matter, with lesions in the left parietal, left occipital, right parietal and right optic radiation, as well

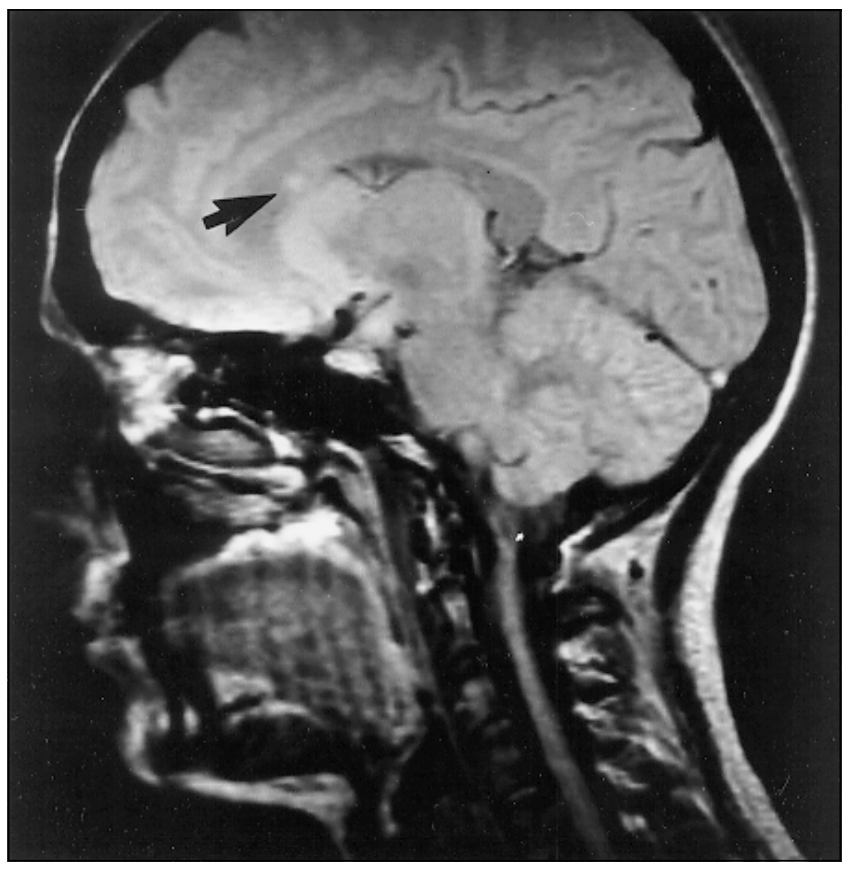

Figure 1) Left Magnetic resonance imaging (MRI) shows axial $T_{2}$ weighted images of $L$ occipital hyperintense subcortical lesion (arrow). Above Sagittal proton density image through the corpus callosum shows a small lesion at the callososeptal interface (arrow). MRI findings strongly support the diagnosis of multiple sclerosis

as a lesion in the corpus callosum (Figure 1). A lumbar puncture revealed normal cells, protein and glucose, and negative bacterial microbiology. However, oligoclonal bands were present, supporting the diagnosis of multiple sclerosis. Somatosensory evoked potentials showed interference with conduction in the left cerebral hemisphere consistent with lesions in the left subcortical white matter.

The patient improved somewhat on dimenhydrinate suppositories and was discharged after eight days of hospitalization. However, she was readmitted with worsening nausea and vomiting six days later; she also complained of diffuse nonthrobbing headaches. She was started on intravenous methylprednisolone sodium $250 \mathrm{mg}$ q6h and intravenous promethazine hydrochloride $25 \mathrm{mg}$ q4h. During this second hospitalization, an episode of right hand and right thigh paresthesia lasted two to three days. A repeat CT scan of the head showed a prominent demyelinating plaque in the left corticomedullary region. She gradually improved and was discharged on oral promethazine hydrochloride 26 days after the second hospitalization. Promethazine hydrochloride was discontinued three months later, without a relapse of gastrointestinal symptoms. She has been well without gastrointestinal or neurological symptoms for the past five years.

\section{DISCUSSION}

Gastrointestinal manifestations of multiple sclerosis include transfer and transport dysphagia (9), nausea, vomiting (2), Brun's syndrome (vertigo, vomiting, headache and visual disturbances with positional change of the head) $(2,10)$, constipation and fecal incontinence $(11,12)$. Inflammatory 
bowel disease has also been associated with multiple sclerosis (13). In the majority of reports gastrointestinal symptoms are described in patients with established multiple sclerosis and most often are accompanied by objective neurological findings. Gastrointestinal work-up is usually negative.

As a primary initial manifestation of multiple sclerosis, nausea and vomiting are distinctly unusual and are most commonly associated with vertigo and eighth nerve involvement. Diarrhea, except in the context of fecal incontinence, is not mentioned as a feature of multiple sclerosis.

The initial diagnostic impression in our patient was that of prolonged gastroenteritis. However, the prominence of nausea was felt to be disproportional to other features. The subsequent gastroenterological work-up did not disclose any plausible explanation for the patient's symptoms. Her family history, amalgamated neurological evaluations, MRI findings (14) and cerebrospinal fluid abnormalities (oligoclonal banding) established that our patient had familial multiple sclerosis. The explanation of her symptoms, however, is less clear. Overt demyelinating plaques were not visualized in the usual centres associated with vomiting. Lesions involving the dorsal motor nucleus of the vagus, the nucleus ambiguous or the autonomic nuclei in the medullary reticular formation are classically associated with vomiting and upper gut motility disturbances $(15,16)$. Lesions in the floor of the fourth ventricle, the area postrema or chemotactic trigger zone for vomiting can lead to similar symptoms (8). It is, however, not an absolute requirement for diagnosis that one of the specific centres described be involved directly. Afferent or efferent fibre connections to these regions may mediate similar symptoms. For example, the lateral tegmentum of the pons and middle cerebellar peduncle have been implicated in the projectile vomiting of a man with metastatic malignant melanoma (16). However, microscopic plaques below the resolution of MRI or CT scanners in relevant ar-

\section{REFERENCES}

1. Percy AK, Nobrega FT, Okazaki H, Glattre E, Kurland LT. Multiple sclerosis in Rochester, Minn; a 60 year appraisal. Arch Neurol 1971;25:105-11.

2. Herndon RM, Rudick A. Multiple sclerosis and related conditions. In: Joynt RJ, ed. Clinical Neurology, vol 3. Philadelphia: JB Lippincott, 1991;13:1-60.

3. Meeroff JC, Schreiber DS, Trier JS, Backlow NR. Abnormal gastric motor function in viral gastroenterology. Ann Intern Med 1980;92:370-3

4. Feldman M, Schiller LR. Disorders of gastrointestinal motility associated with diabetes mellitus. Ann Intern Med 1983;98:378-84

5. Camilleri M, Malagelada JR, Stanghellini V, Fealey RD, Sheps S. Gastrointestinal motility disturbances in patients with orthostatic hypotension. Gastroenterology 1985;88:1852-9.

6. Wood JR, Camilleri M, Low PA, Malagelada JR. Brain stem tumor presenting as an upper gut motility disorder. Gastroenterology 1985;89:1411-4.

7. Szilagyi A, Stern J, Armanious S, Brem S. Gastroparesis secondary to a medullo blastoma of the posterior fossa. Clin Nucl Med 1987;12:864-6.

8. Drachman DA, Diamond ER, Hart CV. Posturally evoked vomiting; association with posterior fossa lesions. Ann Otol Rhinol Laryngol 1977;86:97-101.

9. Daly DD, Code CF, Anderson HA. Disturbances of swallowing and esophageal motility in patients with multiple sclerosis. Neurology 1962;12:250-6. eas cannot be ruled out and may have played a significant role in her symptoms.

In contrast to the dysautonomia commonly observed in diabetes mellitus $(18,19,20)$, the gastric emptying disorder in patients with central causes may respond poorly to prokinetic therapy. The gastric emptying disturbance, both in the present case and in a previously reported patient with prolonged nausea, vomiting and diarrhea secondary to a posterior fossa tumour (7), did not respond to such therapy.

The diarrhea experienced by our patient is more difficult to explain. The presence of edema and spotty inflammatory cells on sigmoid biopsy raised the possibility of an unrecognized infectious agent. However, no agent was found and the upper gut symptoms were much more prominent than diarrhea. We speculate that her diarrhea and upper gastrointestinal symptoms may have been of autonomic origin. The combination of gastric dysmotility and autonomic disturbance has been shown to be correlated in a number of disorders such as primary gastric dysautonomia or irritable bowel syndrome $(21,22)$. As well, constipation in multiple sclerosis may be related to autonomic dysfunction (23).

\section{CONCLUSIONS}

We believe our case represents an unusual manifestation of multiple sclerosis. We base this conclusion on the definitive diagnosis of multiple sclerosis, the absence of specific gastrointestinal disease and the therapeutic response of the nausea and vomiting to primary central acting promethazine hydrochloride and corticosteroids. Furthermore, the absence of any gastroenterological disease, such as inflammatory bowel disease, after more than five years of follow-up also supports our impression. Cases such as these should alert nonneurology physicians that persistent upper and lower gastrointestinal symptoms may, on occasion, be a consequence of primary central nervous system pathology.

10. Alpers BJ, Yaskin HE. The Brun's syndrome. J Nerv Ment Dis 1944;100:115-34.

11. Weber J, Grise P, Roquebe AM, et al. Radiopacque markers transit and anorectal manometry in 16 patients with multiple sclerosis and urinary bladder dysfunction. Dis Colon Rectum 1987;30:95-100.

12. Chia YW, Fowler CJ, Kamn MA, Henry MM, Lemieux MC, Swash $\mathrm{M}$. Prevalence of bowel dysfunction in patients with multiple sclerosis and bladder dysfunction. J Neurol 1995;242:105-8.

13. Wills A, Hovell CJ. Neurological complications of enteric disease. Gut 1996;39:501-4.

14. Gean-Marton AD, Vezina LG, Martin KI, et al. Abnormal corpus callosum: A sensitive and specific indicator of multiple sclerosis. Neuroradiology 1991;180:215-21.

15. Eliasson S. Activation of gastric motility from the brain stem of the cat. Acta Physiol 1953;30:199-214.

16. Parani FD, Norman WP, Gillis RA. Medullary parasympathetic projections innervate specific sites in the feline stomach. Gastroenterology 1988;95:277-88.

17. Baker PCH, Bernat JL. The neuroanatomy of vomiting in man: association of projectile vomiting with a solitary metastasis in the lateral tegmentum of the pons and the middle cerebellar peduncle. J Neurol Neurosurg Psychiatry 1985;48:1165-8.

18. Achem-Karam SR, Funakoshi A, Vinik AI, Owyang C. Plasma motilin concentration and interdigestive migrating motor complex in diabetic gastroparesis: Effect of metoclopramide. Gastroenterology 1985;88:492-9.

19. Koch KL, Stem RM, Stewart WR, Vasey MW. Gastric emptying and 


\section{Szilagyi et al}

gastric myoelectrical activity in patients with diabetic gastroparesis: Effect of long-term domperidone treatment. Am J Gastroenterol 1989;84:1069-75.

20. Janssens J, Peeters TL, Vantrappen G, et al. Improvement of gastric emptying in diabetic gastroparesis by erythromycin. N Engl J Med 1990;322:1028-31.

21. Camilleri M. Disorders of gastrointestinal motility in neurologic diseases. Mayo Clin Proc 1990;65:825-46.
22. Aggarwal A, Gutts TF, Abell TL, et al. Predominant symptoms in irritable bowel syndrome correlate with specific autonomic nervous system abnormalities. Gastroenterology 1994;106:945-50.

23. Camilerri M. Disturbances of gastrointestinal motility and the nervous system. In: Aminoff MJ, ed. Neurology and General Medicine, 2nd edn. New York: Churchill Livingstone, 1995:267-84. 


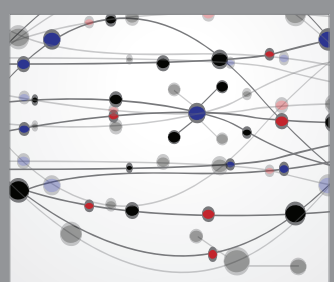

The Scientific World Journal
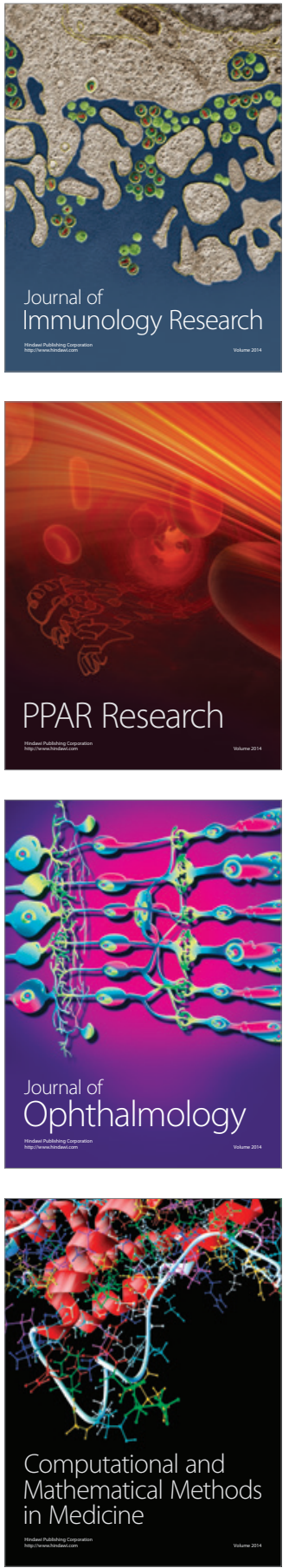

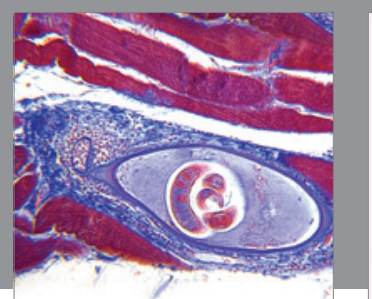

Gastroenterology Research and Practice

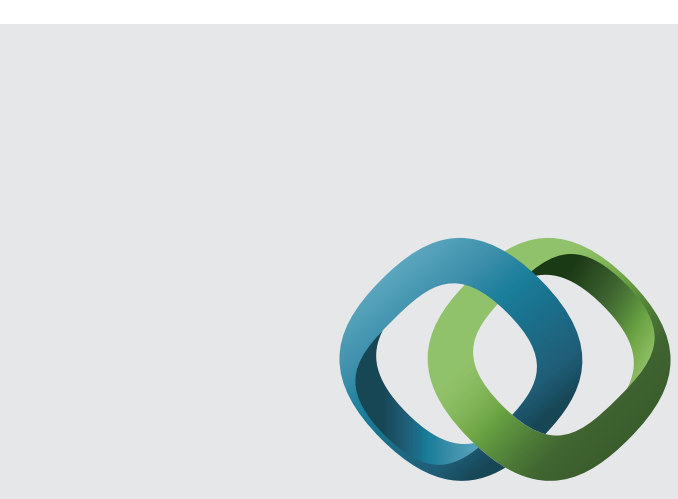

\section{Hindawi}

Submit your manuscripts at

http://www.hindawi.com
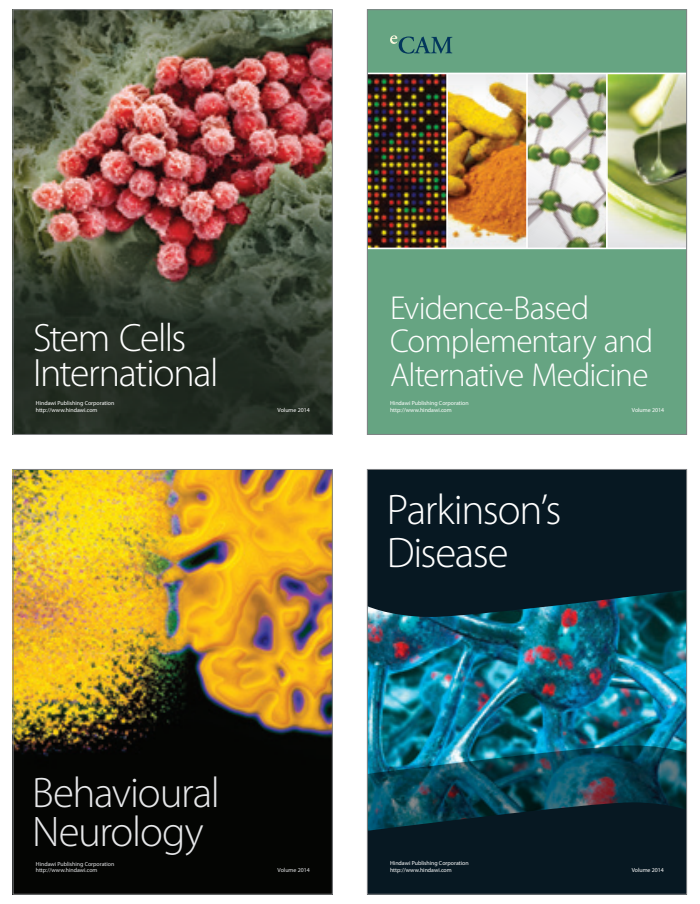
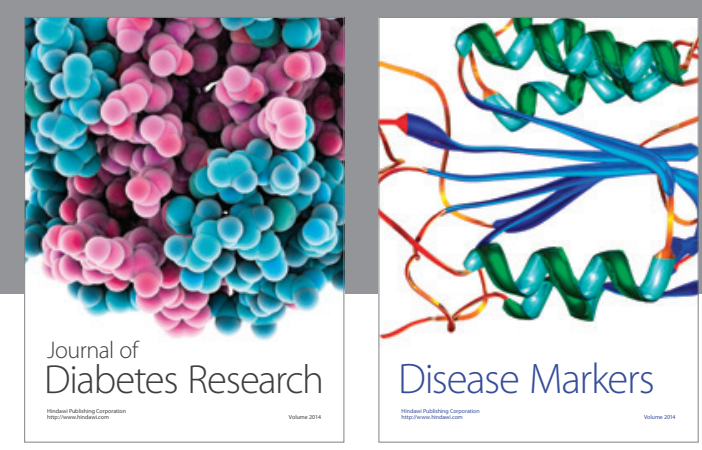

Disease Markers
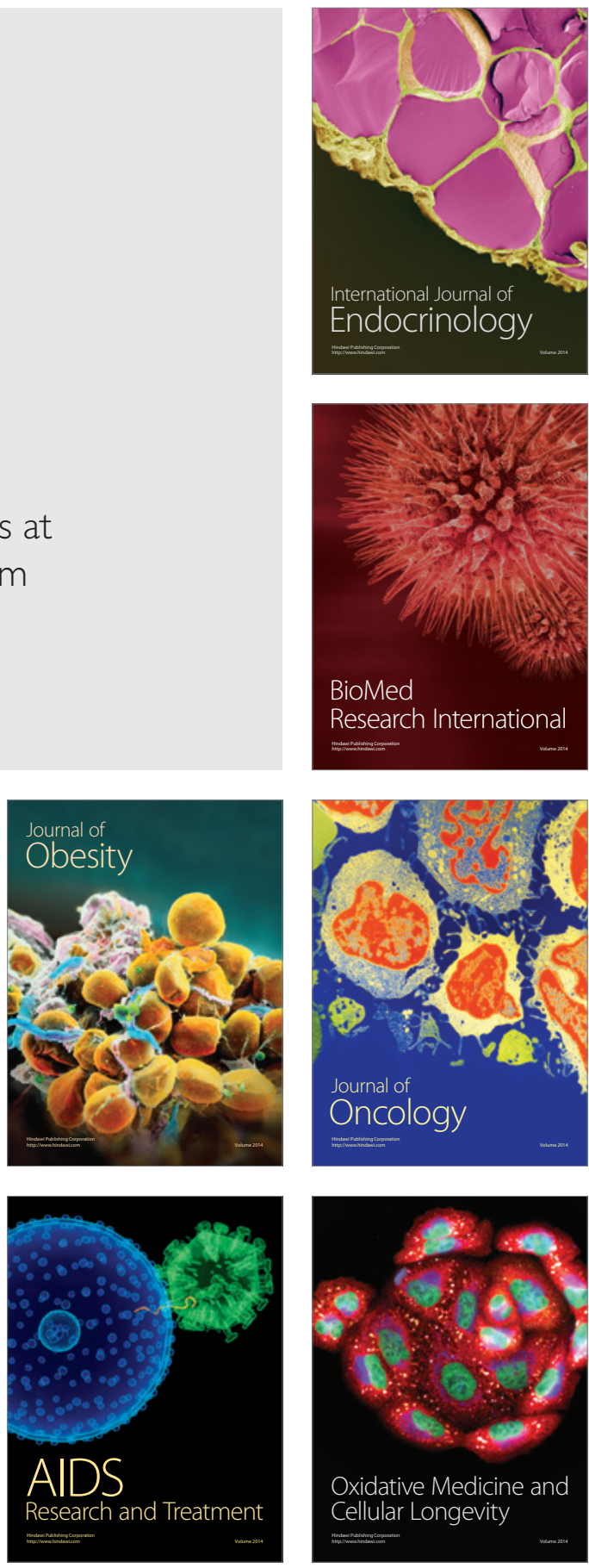